\title{
Analysis of Future Technological Changes in Science
}

\author{
${ }^{1}$ Abirami S K and ${ }^{2}$ Keerthika J \\ ${ }^{1}$ Department of Computer Science and Engineering, Sri Eshwar College of Engineering, Coimbatore, India. \\ 1abirami.s.k@sece.ac.in
}

\author{
Article Info \\ Journal of Computing and Natural Science (http://anapub.co.ke/journals/jens/jcns.html) \\ Doi: https://doi.org/10.53759/181X/JCNS202101016 \\ Received 13 March 2021; Revised form 17 April 2021; Accepted 22 June 2021. \\ Available online 05 October 2021. \\ (C)2021 Published by AnaPub Publications.
}

\begin{abstract}
Rapid technological advancement provides a major potential to fulfill the agenda 2030 and the 2030 Agenda For sustainable development. Technological breakthroughs can help to eradicate poverty, monitor environmental sustainability key performance indicators, enhance food security, promote resource efficiency and effectiveness, enable deep structural transition, support social integration, combat ailments, and enhance access to higher education. Technology advancement also creates new policy problems, threatening to outrun authorities' and society's ability to respond to the changing brought about by new technology. Automation may have an uncertain and possibly detrimental effect on the economy, profitability, internationalization, and competitiveness. In that regard, this paper will focus on the technological changes in the field of science. The paper will start on an analysis of the effect of fast technological development on global disparities, then literature survey before evaluating the technological changes in science.
\end{abstract}

Keyword - Natural Science, Technological Changes, Science

\section{INTRODUCTION}

High-tech development methods may exacerbate disparities, and this dynamic is amplified in low-income nations. Highskilled, high-wage employment is created by new technology-based companies, but they are few and few between. When high-wage workers spend their earnings in the local economy, lower-wage service jobs emerge. Many people in countries with traditionally high levels of education have the required skills for the jobs, and compensation increases gradually. However, in countries with low levels of education, in which only very few people possess the required skills, those who acquire these jobs may also receive hyper-wages, significantly expanding the income disparity at the top. The more the globalization of the sector, the more likely this is to happen. Increased spending will not provide much income to the impoverished when combined with high underemployment, which maintains service wages low.

Economies that benefit from globalization by generating new manufacturing or service employment will witness growing inequalities among towns and cities, and between center and peripheral regions. China exemplifies this trend. It has about the same degree of inequality as the United States. Variations across regions, rather than disparities between individuals, explain considerably more of China's disparity than they do in the US. Technology advancement is almost always everywhere and a city phenomenon, particularly when the process is sped up by a local area network of knowledge, as it is in Bengaluru, India.

Inequalities in technology across nations emerge in industry and commerce, as well as in people' quality of life [1]. The vast disparities in family income throughout the world are strongly influenced by variations in national levels of income. Higher educational levels attained in wealthier nations result in more employment opportunities. Even low-income families in high-income countries may have access to great transit systems, adequate healthcare, and housing that would otherwise be available only to middle-income families in less-affluent countries. Global economic technological development brings both possibilities and dangers to country economies [2]. Middle-income countries may use innovation to their advantage if knowledge is concentrated appropriately and a determined effort is made to apply it to critical industries. Using innovativeness for competition typically requires an active strategy, whether via focused research and development, standards, or public procurement. National champion companies and public procurement rules, for example, helped the GSM sector in the Nordic nations considerably. Nokia, for example, rose to prominence as a worldwide power in an industry that was already gaining traction abroad. National businesses in less prosperous areas frequently choose to acquire the technology they require to upgrade operations, increase productivity quickly, and remain competitive. Importing technologies might be the first step toward technological progress and education.

Nevertheless, because much of the knowledge included in such inventions is tacit and not easily transferable, the importation of technology sometimes does not necessarily result in the development of local capabilities. Finding the means to engage in scientific knowledge is a tough job, and poor educational institutions stymie efforts to attain greater levels of performance. Another opportunity provided by globalization is the rising middle class in developing countries. Upgrading the living standards in developing nations such as Brazil, India and China provides novel markets for cheaper goods tailored for those nations' infrastructure circumstances (e.g., limited or uneven electric power). Firms situated under 
these circumstances are more likely to grab these marketplaces than those designed for wealthy nations' infrastructure constraints.

Because non-wealthy nations have more people than affluent ones, expanding these goods outside the nationwide marketplace may create a novel competitiveness base. While these businesses innovate for conventional reasons, they help a group of people who are just not usually the focus of high-tech enterprises in the global North. This kind of technology has the potential to alter the redistributive patterns of the global economy by shifting market and production power away from the center. Disruptive technology development brings with it both possibilities and risks. Beginning in the 1970s, the advancement of technology demanded a fast development of new industrial capacity. The "Asian tigers" stepped into this potential area and carved out a new niche for themselves. Taiwan Province, China, for example, has emerged as a significant semiconductor production center. On the other hand, some people are concerned that the Fourth Industrial Revolution may remove many of the new employment generated in poor and middle-income nations in recent decades. This is due to the fact that industrial and service occupations are being reintroduced with much smaller worker forces, necessitating higher skill levels.

Whether or whether technological progress enhances the quality of life for a country's people is often determined by regulatory frameworks, absorption capacity, and pricing. Anti-retrovirals for the management of HIV/AIDS, for example, have not been accessibly for a long time because of the high costs for individuals with little means who rely on public health facilities to supply them [3]. However, aggressive application of patent law provisions, as well as a worldwide humanitarian movement, drove down costs. Another example: until the last decade, local farmers in Mozambique were unable to profit from plant tissue culture due to a lack of facilities and qualified personnel, despite the fact that it has been extensively embraced in other nations. The expansion of technical research, production, and distribution skills was aided by a limited amount of international financing and education outside of the nation. Similarly, competition rules aided in the accessibility of pre-paid cellular telephones, allowing smartphones to connect low-income households and paving the door for broadly useful developments such as electronic wallets. In summary, policies that keep disadvantaged people in mind may help share the advantages of emerging technology.

This paper focuses on an analysis of technological changes in the field of science. The rise of the Industrialization, a series of digitalized technologies that may usher in a new era of innovation, illustrates the risks and opportunities of rapid technological change. In that regard, this paper has been organized as follows: Section II reviews the relevant literature texts; Section III presents the critical analysis of the research while Section IV concludes the paper.

\section{LITERATURE SURVEY}

D. Guttieres in [4] conducted an analysis on the rapid technological development and how it has the potential to "leave no one behind." Some of the connections between Industrial revolution 4.0 and inequality are expected to be familiar from previous technological revolutions, while others are likely to be unique to this convergence. Both are highlighted by the dynamics discussed in this chapter.

S. Shane in [5] argues that the new technology regimes may seem to be dangerous. This is particularly true at a time when technological visionaries are painting the future in the most pessimistic light imaginable. Several of the most drastic changes never happen or have their consequences controlled by the time they do, while other major alterations have an effect on societal structures that were not anticipated at the start. Second, just as past emergent technologies grew, developed, and spread in unexpected ways, Industrial revolution 4.0 would grow, develop, and disseminate in unpredictable ways when innovations fade away. Entrepreneurs will find new uses for goods and processes to accomplish a variety of goals. The more disruptive the techno-economic paradigm's course, the more unexpected the applications will be.

V. Chang, G. Wills and P. Baudier in [6] argue disruptive impacts of Industry 4.0 have already been seen in many industries, including retail (for example, Amazon) and transport (for example, Uber). Additionally, there is no indication that the new hybrid professions, which rely on both human platforms and mobile linkages, are being well rewarded among ride-hailing drivers. Third, there are probably to be gender differences in the effects. Women-dominated professions, such as the day support and home service, have been recognized as being less susceptible to robotic displacement. However, declaring that males would be the future's vulnerable group is certainly premature, particularly given the gender divisions produced by women's underrepresentation in STEM work families.

J. Hinzpeter in [7] posits that the informal sector is unlikely to be affected by robots and artificial intelligence; in fact, it may expand if the costly new technology boost rates in the official economy. Based on regional, rural and urban distribution, particular applications (such as robotic systems in manufacturing) are more location-based, whereas others actually happen in cyberspace (such as data-based marketing). Instead of increasing geographical centralization, the latter may lead to an increase in spatial decentralization.

C. Bambra in [8] argue that new systems will be required to combat inequalities and rebuild social cohesiveness, just as they have been in past technological revolutions. Just as the spatial preponderance of employees enabled organizing and unionization during the industrial revolution, social media may make it easier to organize dispersed workforces. Universal basic income plans may be used to create public social safety nets. Activist investors may change corporations such that social responsibility is a key role. New uniting factors may arise when new centripetal forces intensify polarization.

In [10], D. Schartinger, D. Wilhelmer, D. Holste, and K. Kubeczko draw the conclusion that legislators will have to formulate a strategy regarding technical forethought and assessment of technology's destabilisation implications over years, if not generations, if communities are to better cope with quick clip and increased context of technological advancement. Forecasting is about putting together key drivers of transformation and areas of information to develop strategic views and 
insights to affect the future. Emerging technological forethought abilities (e.g., horizon surveillance and externally imposed implications studies) can assist nations in recognizing and utilizing the potentials of engineering areas for continuous production, technology management concepts in the quick, intermediate, and distant future, and considering the potential role of technological advancements. There are also major implications for the kind of facts and techniques needed to support policy formulation.

O. Popova in [10] evaluate on combining methods and data for technical, economical, societal, and ecological impacts, for instance, in an evaluation of innovation's ecological effects. Member states of the United Nations are increasingly realizing the value of foresight efforts in assisting communities and policymakers in adapting to the changes brought about by the development of new technology. Technology and evaluation exercises, according to the Economic and Social Council, may assist decision makers and partners in implementing the 2030 Agenda by identifying problems and opportunities that can be strategically addressedThe Council has encouraged countries to undertake systematic research for forecasting exercises on emerging trends in STI in ICTs in the most current declaration on STI for development. They also considered strategic foresight efforts on regional and global issues at the national level, as well as their effect on development, given the context of the united Nations sustainable Development Goals.

S. Talmon argues in [11] that recent General Assembly conclusions on STI for exploring the impact of digital disruption on achieving the SDGs motivate Member States to recommence to examine the effect of digital disruption and to conduct technology assessment and forethought activities to evaluate their development potential and mitigate potent threats. Countries may look at methods to undertake technological evaluations and forecasting projects on a national, regional, and worldwide level.

C. Po-ying argues in [12] that if there would be an intergovernmental body to evaluate such developments and figure out their implications for low - income and middle-income countries, national decision-makers' ability to respond would be significantly affected. International attempts to aid national responses, on the other hand, must rely on a broader set of ideas and data than international organizations can offer. Different research and development groups e.g. the Society for the Study of Research and Innovation (SSRI), are dedicated to evaluating trends and providing possibilities for action in Europe, North America, and wealthy Asia.

International and domestic organisations may assist in the development of this monitoring capacity inside low- and middle-income nations by supporting a broader variety of scientists, both for joint development projects and travelling to meet their academic peers. In both the long and short term, Ph.D. schools offer a notably cost-effective method of developing knowledge. Relationships to policymakers are critical for such research to be helpful, and holding frequent policy or scholarly exchanges would assist guarantee that information is pertinent, valuable, and available. Section III below focuses on the rapid technological changes in the field of science.

\section{CRITICAL ANALYSIS}

Key Messages

Advancement of technology has the capacity to exacerbate existing national and international divisions, as well as create ethical security and privacy issues, cybersecurity, data governance, and protection. Although the processes of globalization of technology development have the potential to widen socioeconomic disparities, governments may encourage investments that broaden skills and promote innovation among and for marginalized populations. Building and maintaining successful innovation systems is part of national plans for leveraging fast technological change for long-term development. Tackling the education-employment connection, creating endogenous innovation capabilities, developing digital competencies to close digital inequalities, and improving technological foresight ability are all important policy concerns. Governmental and multi-stakeholder initiatives to improve digital capabilities may help refocus and enhance international collaboration on frontier technologies innovation [13]. Educational, technological, industrial, and civil society efforts, as well as UN system-wide operations, may assist guarantee that rapid technology progress does not leave anybody behind.

Science and engineering are profoundly ingrained in the business processes that aggravate disparities within and between nations in both developed and developing economies. While scientists and engineers react to these initiatives in their respective areas and nations, they are also members of worldwide communities that are ultimately focused on the public good. Many of them reside in communities that recognize that fulfilling their intergovernmental obligations helps the global economy, as well as their own. More of the activity of wealthy nations' scientific and technological communities has to be directed toward the needs of people on the edges if they are to assist efforts to reduce disparities. Individuals and organizations make a variety of commitments to this aim. With the help of private funders, academic research institutes adopt this approach on their own. The University of California, Berkeley, for example, has a Global Health Alliance that involves student exchanges as well as collaborative research initiatives with colleagues in Uganda, South Africa, South Africa, Nicaragua, Mexico, India, China and Brazil.

The MIT CoLab (Community Innovators Laboratory) is a research \& design facility that concentrates on creativity from the periphery, serving as a research \& design centre that taps into the wealth of knowledge in underprivileged communities to address inequality issues. There are many worldwide initiatives using the Internet of Data analytics and artificial intelligence that are aimed at developing capability [14]. For instance, the collaboration between the Abdus Salam International Centre for Theoretical Physics (ICTP) and African Center of Excellence in Internet of Things (ACEIoT) has resulted in the creation of an Internet of Things Doctoral Program. With the goal of promoting diversity in AI, the online learning portal Fast.ai provides free deep learning courses. The platform has introduced deep learning diversity and international fellowships, giving participants access to cutting-edge AI training. 
Technological Changes in the Field of Science

The announcement that the LIGO group had established a gravitation wave enthralled the scientific world in 2016 . The Laser Interferometer GravitationalWave is a US National Science Foundation-supported joint project run by Caltech and MIT. LIGO is the step in a series of breakthrough astronomical breakthroughs, such as the capacity to visualize cosmos through the radio wave and the gamma radiations or to identify cosmic neutrinos and rays. A new technological advancement seems to have the potential to unlock a window on space, culminating in major novel hypothetical breakthroughs. These, in turn, may influence future technical advances. This interaction of technical and basic theoretical advancement can be seen in all natural disciplines, including computer science, we suggest. Theoretical representations of real-world computing environments were used to construct some of the earliest computer models. These were, for example, human 'computers' doing calculations for the Turing Machine. As new desktop computers, spanning from quantum states to DNA processor, and even massive systems of human "social computers" reach a critical stage of development, they demonstrate how technical solutions may spur the development of conceptual models and computing tools.

As much as astrophysics is about telescopes, so is computer science. As Moore's Law, the formerly unstoppable growth in computer power, runs out, non-standard and unorthodox technological solutions have gained popularity. While multiprocessor and parallel conventional techniques allow for some improvements without increasing transistor densities, there is a developing agreement that the next important advancement will come from innovations outside semiconductor technology and binary logic. The European Commission has proposed a $€ 1$ billion quantum technology flagship, with Google \& NASA, and a number of universities and state laboratories, doing active research and utilizing quantum computing.

Data encoding and decoding in DNA molecules, neurosilicon hybrids device and biologically-inspired neural and controlling activities of the slime mold are all examples of biological computing being created. The internet's massive advancement has allowed "social machines"—Galaxy Zoo, protein FoldIt, Wikipedia, and a slew of citizen science toolsto do calculations that aren't possible with current silicon-based technologies alone. All of these gadgets, from the futuristic to the mundane, have one thing in common: they are presently outside the scope of traditional computation. The standard silicon technology is constructed on the framework of hypothetical ideologies and techniques that range from lambda calculi via coding, translation, and validation. The new technologies do not seem to have much access to these tools.

How can a slime mold be programmed? When it comes to protein folding, what is the assembler? In a social machine, how do you assemble for a person? Technological advances may be continuous, stochastic, non-linear, temperature-based, sloppy, complex and symbolic computational compression algorithms and decoding, and one-shot invention and performance, among other things. We believe that these technologies' information-processing capabilities will be underused until we can define and quantify how or when computation happens in these platforms, and then integrate or build the full set of different computer engineering methods. We think that an expanded computer science is required to unleash the potential of these systems by treating them with practical and theoretical rigor, as well as combining them with current technology to create extensible and multifunctional devices.

Abstract Turing Computers and similar calculi have traditionally been used to conceptualize and advance computer science. The von Neumann stored programme architecture realizes this discrete, symbolic, logical, deterministic underlying paradigm in a similar but distinct way. Other theories of computing, such as Shannon's mostly forgotten GPAC mathematical computation, which is based on the technologies of divergent analysers, have tended to be neglected as a result of this technology's success and widespread use. As a consequence of choosing just one model, standard techniques of computing abstract away the physical implementation, resulting in applied mathematics that is often considered as a discipline of arithmetic instead of a theoretical physics expressed in mathematical formalism.

Due to its lack of connections to the real natural equipment, this conceptual framework could be rendered at a loss whenever encountered with non-standardized computer models. The typical approach is to potentially impose a convectional bit and a logic-gate framework from the top-down considering that it is an effective approach of computing. For instance, the circuits that are delicate in quantum algorithm may be made to behave like ordinary bits following classical logic. When these gadgets are permitted to function as indigenous "qubits," or quantum states, with their own computational logic gates, they acquire their true potency. Imposing the standard computing architecture on many nontraditional computers is as inefficient (or impossible) as using a powerful optical telescope in order to effectively detect cosmic neutrino. It is not considered that pushing unusual systems into traditional computing paradigms will allow them to reach their full potential.

Nontraditional programming has gone too far in the other way, viewing computers as just a consequence of physics, chemistry, or biology, whereas conventional computer science sees itself as a subject of mathematics. Arguments regarding processing capacity often focus on the gadget's physical theory rather than what the device can actually do. Modeling neural nets using real-valued convolution layers, for instance, has led to assertions that all these systems can calculate actual figures to arbitrary precisions exceeding the abilities of the general-purpose PC systems.

Nonetheless, these complete abstract, valued accuracies are entirely beyond the device's physical capabilities: it cannot be seen or utilized. Computing concept must not be enforced from the top down without considering the device's physical theory: computer science is not arithmetic. Computer science is not considered as physics, and the computational capacities of systems are unnecessarily the same as the computational capabilities of the hardware theory. So, what exactly is it? We think it combines the best of both worlds, including the intricate interaction of mathematical and physics theory through a critical relationship: representation. Mastering computers is a prerequisite for studying computer science.

Computers bridge the existing gap between abstracts world of digital signal processing and the actual world of transistors, classical particles, and biomolecules in a form that can be accurately described. Observe section (a) of Fig 1, in which a 'compile loop' begins with a theoretical task like adding two integers, identifying prime numbers, or identifying the 
shortest route. The information is usually represented in a high-level vocabulary and then translated into the device's native tongue. In simple terms, this embedding is still an intellectual process: the computation's representation has been translated from one vernacular to the other. The targeted physiological gadget is now connected to the computer, and the native tongue input is created. The machine is set up, and the computer's mechanisms are started. The system then operates as a physical phenomenon with an output energy in its complete form. To determine the computation's outcome, we must first determine to which intellectual state the physiological one coincides: which programme state is conveyed by the physiological state of the device? The abstract form result is then (reflectively) translated into a vernacular to address the initial issue.

The remedy was generated by the machine. The goal of the computation phase, if a system is efficient and runs without mistakes, is to concurrent actual and conceptual behaviors. The resolution is an ideal response to an ideal issue; if the software could be "executed" fully abstract ideas, the response might be discovered without involving any gadget in the process, be it a machine or a ballpoint pen based approach simulation. For this conceptual translation, machines are utilized as a practical surrogate. A good system is built in such a way that allowing actual phenomena to operate parallels the agency's abstraction behaviour. A computer is a machine that solves problems by manipulating the physical manifestation of abstract notions. It's not the same as a calculation: a calculation is conceptual, but a machine is actual, and the two are linked by (considerable) representations and implementation. This importance of depiction lies at the heart of the researchers' new framework, "Abstraction/Representation Theory (AR theory"). The concept is an instrument for the elements of computer science and beyond, including drawings in Fig 1, portion (a) of the image shown and an accompanying equilibrium isotherm framework.

The complicated interaction of arithmetic, physiological science and representations isn't limited to calculating. It also powers the process for putting conceptual hypotheses to the proof in the scientific method. For example, we may draw a graph depicting the relationship between astronomia and observatories, with key parallels and contrasts to computers. The Fig 1 part (b) depicts the relationship between experiment and theory in the LIGO experiment. The theoretical and practical worlds are once again concurrent, but this time the study begins with the practical instrumentation rather than an embedding of an ideal computing issue. In the concept of gravity waves, the equipment has an abstract model: it can sense gravity waves. There is a conceptual assumption about how the equipment will function if such waves exist in the abstraction domain as well. If the research succeeds, as it did with LIGO, the hypothesis and the abstraction understanding of the actual result will match up to a certain standard deviation. The state of the system can be eliminated entirely if a concept is reasonably accurate: abstraction concept can anticipate physical phenomena. We may deduce a profound fact from these two images.

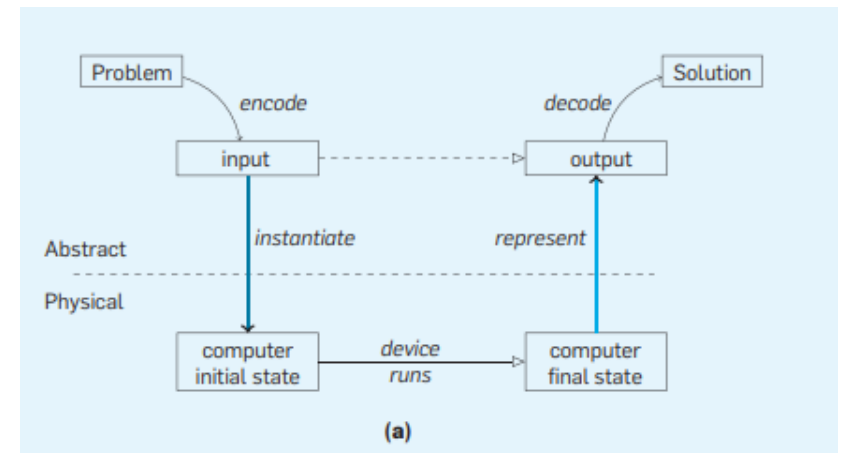

Fig 1a. The equilibrium isotherm framework.

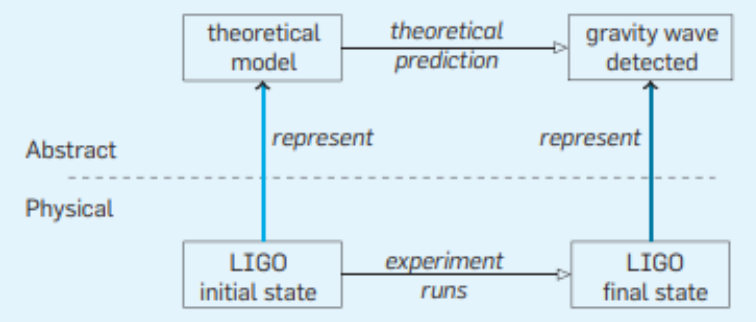

(b)

Fig 1b. The relationship between framework experiment and theory in the LIGO experiment

In the same way that a mathematical formalism may forecast physical activity, the physical behavior of an unit in a system can be used to 'forecast' the outcome of an abstracted calculation. Scientific method and computation are intrinsically connected; the connection is technological. In the two drawings, pay attention to the location of the representational arrows. They only go one way in an investigation: this is the abstraction model's depiction of physical processes. Another sort of connection exists in computer technology: the implementation of abstract principle in physical 
processes. Implementation is more difficult than modeling because it necessitates the construction of a mechanism that, when represented, yields the abstraction specifications to be implemented; this modeling, in turn, necessitates a proper scientific grasp of the program's characteristics. Not all abstraction concepts that may be conceived can be literally implemented or indicate something else in the real world ("the current monarch of France") (faster-thanlight travel). A pc is a highly designed instrument, similar to an observatory.

LIGO underwent extensive testing of its different components before researchers were satisfied that it could discover gravitational waves. It may now be used as an observatory to see the cosmos in respect of those waves when the trials are completed. Pcs, too, need construction before they can be used for arithmetic: we must be certain that their physical reaction matches that of the abstraction system in order for the machine to be used to anticipate its conclusion (there can be engineering bugs in hardware). Pcs begin with computer engineering: experimentation on innovative substrate and revolutionary methods of computation.

A new phone can only be built to perform a computation after that process has been completed and we have enough knowledge on how the system functions. What else does AR concept imply for our understanding of computer science? We propose that we now have a method to understand how computational reasoning is generated from a potential computational platform's spatial shape, and how this structure differs significantly among substrate types. Additionally, computer programming encompasses the testing and construction stages of computation, as well as its ultimate application in computers installation. This knowledge instructs us to utilize an empirical and technical approach when building new formalized computer engineering models and techniques for our smart technologies, similar to how new products and instrumentation are developed to address novel phenomenon in the whole of the scientific method.

A program's computing process emerges, but we isolate far from certain devices to a formalized definition. Instead of putting a one-size-fits-all paradigm of computing upon any prospective computer systems, designing these new machines becomes a question of searching for the system's inherent internal operational reasoning. Rather of imposing the mechanism of typical logic gates from the top down, we should examine the system's natural behavior and what "entrances," "sub - routines," and "real concern" it is naturally capable of. We can unlock the real potential of atypical device by separating fundamental computing logic from their physical parts. The fact that we can use our physical interpretation of a platform to guide a computer reasoning does not indicate that this is the only logical that can be used. Other non - standard devices may be able to support various computational methods, much as a computer model may function in either classical or conventional mode. This may be seen across the physical sciences: in thermodynamics, for example, a system can be represented as a flowing fluid or as a group of suspended particles.

With so many alternative computational models of a phenomenon under examination, the goal is to isolate the ones that are useful, innovative, and superior to other substances then utilize that computation concept to design our next generations of pcs. After that, we'll be able to go even further. We will then have a conceptual vocabulary in which to characterize the physical processes themselves, even outside of a particularly computational device, if we had an abstraction computer terminology that described the natural behavior of unusual gadgets. Computer programming might then offer increased logical process terminologies for physicists, biochemistry, and genetics even for opportunities offered, thanks to the interaction of sociable machinery. We trust this will be immediately useful to scientists working in those fields, allowing them to explain the elevated operation of complicated systems and discover new and unexpected linkages between different systems and situations. These processes technologies have the potential to revolutionize the scientific process in the same way that they have revolutionized computer programming.

\section{CONCLUSION}

Computers have a long history dating from the era of controllers and radeon cards. Computer programming is currently spreading out into new fields as a result of the emergence of unique computing systems. As seen from this paper, the real nature of computer science is becoming exposed as the field matures and one computational paradigm no longer suits all. Computer science, like astronomy, may use abstract language to explain physical systems with predictive capacity, advancing the twin interaction of technological and conceptual progress. New computers may help us comprehend the material reality around us by informing new computational ideas. A natural science, such as computer science, would exist.

\section{References}

[1]. Kruglianskas and H. Thamhain, "Managing across nations. Technology-based projects in multinational environments", IEEE Potentials, vol. 19, no. 2, pp. 3-8, 2000. Doi: 10.1109/45.839648.

[2]. G. Mitchell, "Global Technology Policies for Economic Growth", Technological Forecasting and Social Change, vol. 60, no. 3, pp. 205-214, 1999. Doi: 10.1016/s0040-1625(98)00044-4.

[3]. T. Kredo, N. Ford, F. Adeniyi and P. Garner, "Decentralising HIV treatment in lower- and middle-income countries", Cochrane Database of Systematic Reviews, 2013. Doi: 10.1002/14651858.cd009987.pub2.

[4]. D. Guttieres, "An Intra- and Inter-generational Lens into Rapid Technological Change", Development, vol. 62, no. 1-4, pp. 37-42, 2019. Doi : 10.1057/s41301-019-00220-5.

[5]. S. Shane, "Technology Regimes and New Firm Formation", Management Science, vol. 47, no. 9, pp. 1173-1190, 2001. Doi: 10.1287/mnsc.47.9.1173.9785.

[6]. V. Chang, G. Wills and P. Baudier, "Impacts and investigations of disruptive technologies for Industry 4.0", Technological Forecasting and Social Change, vol. 174, p. 121232, 2021. Doi: 10.1016/j.techfore.2021.121232.

[7]. J. Hinzpeter, "About Artificial Intelligence: Robots and Philosophy", International Journal of Biochemistry \& Physiology, vol. 4, no. 2 , 2019. Doi: $10.23880 /$ ijbp-16000148.

[8]. C. Bambra, "First do no harm: developing interventions that combat addiction without increasing inequalities", Addiction, vol. 113, no. 5, pp. 787-788, 2018. Doi: 10.1111/add.14116.

[9]. D. Schartinger, D. Wilhelmer, D. Holste and K. Kubeczko, "Assessing immediate learning impacts of large foresight processes", Foresight, vol. 14, no. 1, pp. 41-55, 2012. Doi: 10.1108/14636681211210350. 
[10]. O. Popova, "Developing The Methods For Evaluation Of Economic Efficiency Of Ecological Investments", Economical, vol. 2, no. 221, pp. 151158, 2019. Doi: 10.31474/1680-0044-2019-2(21)-151-158.

[11]. S. Talmon, "The Legalizing and Legitimizing Function of UN General Assembly Resolutions", SSRN Electronic Journal, 2014. Doi: $10.2139 / \mathrm{ssrn} .2509719$

[12]. C. Po-ying, "How students react to the power and responsibility of being decision makers in their own learning", Language Teaching Research, vol. 11, no. 2, pp. 225-241, 2007. Doi: 10.1177/136216880607074613.

[13]. J. Cutcher-Gershenfeld, "Intraorganizational Bargaining in Multi-Stakeholder Initiatives", Negotiation Journal, vol. 31, no. 4, pp. 393-400, 2015 Doi: $10.1111 /$ nejo.12115

[14]. S. Ogawa and K. Pongtanalert, "Exploring Characteristics and Motives of Consumer Innovators: Community Innovators vs. Independent Innovators", SSRN Electronic Journal, 2012. Doi: 10.2139/ssrn.2188928. 